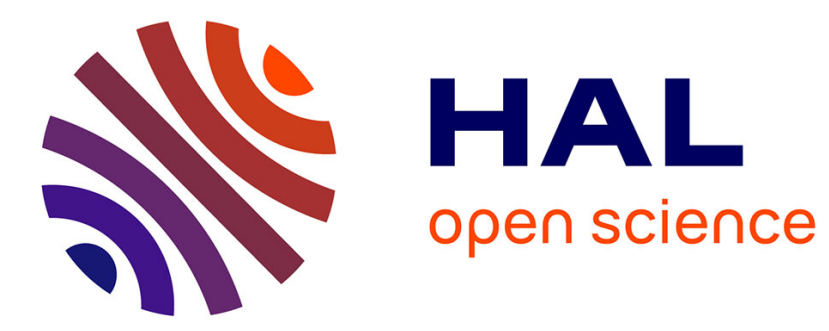

\title{
Minimizing the inter-vehicle distances of the time headway policy for platoon control on highways
}

\author{
Alan Ali, Gaëtan Garcia, Philippe Martinet
}

\section{To cite this version:}

Alan Ali, Gaëtan Garcia, Philippe Martinet. Minimizing the inter-vehicle distances of the time headway policy for platoon control on highways. ICINCO13 - 10th International Conference on Informatics in Control, Automation and Robotics, Jul 2013, Reykjavík, Iceland. hal-02462069

\section{HAL Id: hal-02462069 \\ https://hal.inria.fr/hal-02462069}

Submitted on 31 Jan 2020

HAL is a multi-disciplinary open access archive for the deposit and dissemination of scientific research documents, whether they are published or not. The documents may come from teaching and research institutions in France or abroad, or from public or private research centers.
L'archive ouverte pluridisciplinaire HAL, est destinée au dépôt et à la diffusion de documents scientifiques de niveau recherche, publiés ou non, émanant des établissements d'enseignement et de recherche français ou étrangers, des laboratoires publics ou privés. 


\title{
Minimizing the inter-vehicle distances of the time headway policy for platoon control on highways
}

\author{
Alan ALI ${ }^{1,2}$, Gaetan GARCIA ${ }^{2}$ and Philippe MARTINET ${ }^{1,2}$ \\ ${ }^{1}$ IRCCYN, ${ }^{2}$ Ecole Central de Nantes, rue Noë, Nantes, France \\ Alan.Ali@irccyn.ec-nantes.fr,Gaetan.Garcia@ec-nantes.fr, Philippe.Martinet @irccyn.ec-nantes.fr
}

Keywords: $\quad$ Platoon, control, time headway, constant spacing, string stability, inter-vehicle distance, highways.

\begin{abstract}
Heavy traffic on highways requires the optimization of inter-distances between vehicles in order to reach time performance and to provide safety solution in transport. Variable spacing and constant spacing are the two policies for the longitudinal control of platoon. Variable spacing doesn't require a lot of data (position, speed...) from other vehicles, and string stability using only on-board information is obtained. However, intervehicle distances are very large, and hence traffic density is low. Constant spacing can offer string stability with high traffic density, but it requires at least data from the leader. In this paper, a novel expression of the variable spacing policy has been proposed. It is effective to decrease the distance between the cars, to become nearly equal to the constant spacing policy. It also enables increasing the string stability and the robustness of the control regarding to unmodeled lags, and it can avoid control torque saturation. This novel approach doesn't require heavy communication between the cars. The new control law has been evaluated by simulation with perfect system using Matlab, and with imperfect system using TORCS. The good results have demonstrated the effectiveness of the novel approach.
\end{abstract}

\section{INTRODUCTION}

Nowadays, traffic congestion, pollution, and people safety problems become more and more important due to the explosion in the number of cars.

Treatments of these problems on highways differ from those in urban areas. In highways environment and normal condition, we usually travel at high speeds, cars move faster, the roads are less curved and there are less obstacles compared with the urban areas.

Some proposed ideas impose some changes to the infrastructure (automatic speed limits, roads monitoring, reversible lanes...). Others ideas depend on the automated vehicles to increase the traffic density and to avoid the oscillation. Driving in platoon has many advantages, It increases traffic density and security, at the same time it decreases fuel consumption and driver tiredness(Ricardo, 2009).

From the modeling and control point of view, it is possible to decouple the longitudinal behavior from lateral one, considering that road have low curvature, or using techniques like chained system theory (Thuilot and Martinet, 2004). Lateral control can be done using different modalities like 3D laser (like it is used in the famous Google car), magnetic markers
(PATH project), vision sensor (Royer et al., 2005)...So in highways environment, it is common to concentrate on longitudinal behavior including modeling and control.

Platoon models can be found in (Swaroop, 1997) any communications to full communications between all the vehicles. Others has modeled the platoon using physics-inspired models,(Franck et al., 2004) treated the platoon as multi agents system, the agents (vehicles) interact according to physical phenomena or mimicking animal interaction behaviors, others modeled the interaction as virtual spring-dumper (Yanakiev and Kanellakopoulos, 1996), or as Newton forces (Khatir and Davison, 2004).

String stability means that the errors must not amplify as they propagate through the platoon, and the errors must have the same sign to avoid collision, The definition is given in the time domain in(Swaroop, 1997) and in frequency domain in(Rajamani, 2006).

Local control depends on data from adjacent vehicles, while global control depends on data at least from leader. In local control, the car is totally autonomous, it does not require sophisticated sensors, and can be used in all environments, but trajectory tracking and inter-vehicles distance keeping is not very accurate. In the other hand the global control are 
more accurate, but it needs more sophisticated sensors, and sometimes it needs adaptation in the environment where we use it, and finally it needs very reliable communication systems.

The spacing between vehicles differ according to two control policies (Constant spacing and variable spacing). Variable spacing, usually, doesn't require a lot of data from other vehicles, whats more, it can ensure string stability just by using on-board information (Ioannou and Chien, 1993), but the inter-vehicle distances is very large (function of velocity), and hence traffic density is low. Constant spacing can get string stability with high traffic density, but it requires inter-vehicle communications.

Constant Time Headway (CTH) is the simplest and most common variable spacing policy (Swaroop and Rajagopal, 2001; Yanakiev and Kanellakopoulos, 1996). Variable time headway can vary linearly with the velocity, with relative velocity (Yanakiev and Kanellakopoulos, 1995), or even with the vehicle dynamics and the road conditions (Huppe et al., 2003).

In this work, we will concentrate on the longitudinal control of platoons in highways. We will propose a new modification to the time headway policy, develop the corresponding dynamic control law, study the stability and the control robustness of the platoon and demonstrate the effectivness of the novel approach. The new control law will be a mixture of local and global decentralized control. Security will not be discussed in this paper.

The paper is organized as follows. Section 2 will describe the vehicle and platoon models. The control, string stability, and control robustness will be presented in section 3. Section 4 will explain the simulations results obtained with Matlab and TORCS (The Open Racing Car Simulator) (Onieva et al., 2009). Finally, section 5 will discuss the most important advantages of the proposed approach, and we will make a comparison with other existing approaches.

\section{MODELING AND CONTROL}

As we are dealing with highways with small curvature, in this paper we will only address the longitudinal control:

\subsection{Longitudinal Dynamic Model of the Vehicle}

According to Newton's law, we can write the dynamic equation (Sheikholeslam and Desoer, 1989) of the i-th vehicle in the platoon shown in figure (1) as:

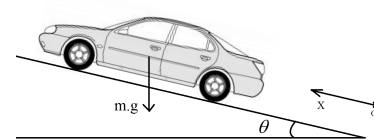

Figure 1: i-th car

$m_{i} \ddot{x}_{i}=F_{i}+F_{i, g}+F_{i, a e r o}+F_{i, d r a g}$

$m_{i} \ddot{x}_{i}=F_{i}-m_{i} g \sin (\theta)-\frac{\rho A_{i} C_{d i}}{2} \dot{x}_{i}^{2} \operatorname{sgn}\left(\dot{x}_{i}\right)-d_{m i}$

which can be represented in figure (2):

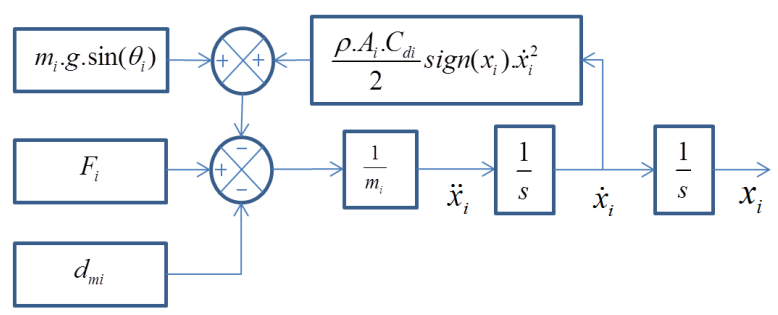

Figure 2: dynamical car model

where:

- $x_{i}$ : Position of the $\mathrm{i}$-th vehicle in $\mathrm{X}$ axis.

- $F_{i}$ : Force produced by the i-th vehicle engine.

- $F_{i, g}, F_{i, a e r o}, F_{i, \text { drag }}$ : Gravitational, aero dynamical and Mechanical drag force respectively.

- $g$ : Acceleration of gravity.

- $\theta$ : Angle between the road surface and a horizontal.

- $\rho$ : Specific mass of air, $A_{i}, C_{d i}$ Cross-sectional area and drag coefficient of the i-th vehicle.

- $d_{m i}$ : Mechanical drag of the i-th vehicle

We can use exact linearization to linearize the previous system to obtain a linear model of the car longitudinal dynamics by taking:

$$
F_{i}=-F_{i, g}-F_{i, \text { aero }}-F_{i, d r a g}+m_{i} u_{i}
$$

Then, we get : where $u_{i}$ is the new command input for the linearized system show in figure (3).

$$
\ddot{x}_{i}=u_{i}
$$




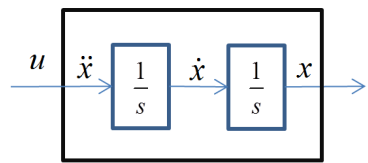

Figure 3: Linearized car model

\subsection{Platoon definitions}

Figure (4) shows a platoon which consists of $N$ vehicles moving at the same speed $v_{d}$ with a desired inter distance $L$ between each set of two vehicles. The leader of the platoon can be driven by a human or autonomously. The followers are controlled to maintain a desired inter-distance.

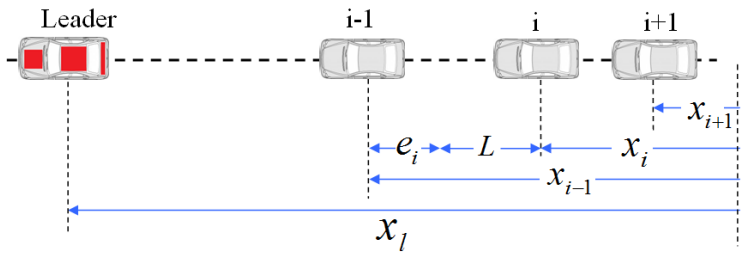

Figure 4: platoon

We define the constant spacing error of the ith vehicle by:

$$
e_{i}=\Delta X_{i}-L a
$$

where

- $\Delta X_{i}=x_{i-1}-x_{i}$ : real spacing between i-th car and its predecessor (i-1-th car).

- $x_{i}$ : position of $\mathrm{i}$-th vehicle.

- $L a=L+D_{\min }$ : actual desired distance.

- $L$ : desired inter-vehicle distance (bumper-tobumper).

- $D_{\text {min }}:$ minimum distance between two vehicles to prevent bumper-to-bumper collision, equal or bigger than vehicle's length.

In the following, we will assume a point mass model for the vehicles and we will ignore $D_{\min }$, so $e_{i}=\Delta X_{i}-L$. The kinematic evolution of the spacing error is given by :

$$
\dot{e}_{i}=\dot{x}_{i-1}-\dot{x}_{i}=v_{i-1}-v_{i}
$$

where $v_{i}$ represents the velocity of the i-th vehicle.

\section{PLATOON CONTROL AND STABILITY}

\subsection{Control Objectives}

The main objectives of the control law are to:

1. Keep the inter-vehicle distance equal to $L$, and to make all vehicles move at the same speed so $\dot{e}_{i}=0$.

2. Assure the string stability of the platoon (the spacing error does not increase as it propagates through the platoon).

3 . Increase the traffic density.

4. Keep the system stable in case of total loss of communication.

5. Accommodate the instability produced by the actuation and sensing lags (robustness).

\subsection{Control Law}

In constant spacing control, the control law will make $e_{i} \rightarrow 0$ so the inter-vehicle distance will become equal to $L$, but this requires, at least, information from the leader to assure the string stability of the platoon and robustness.

In time headway policy, a new term is added to the previous error, which will eliminate the communication with the leader and increase the string stability. A new spacing error is defined as :

$$
\delta_{i}=e_{i}-h v_{i}=\Delta X_{i}-L-h v_{i}
$$

In this case the control law makes $\delta_{i} \rightarrow 0$, so the steady state of the inter-vehicle distance will be equal to $\Delta X_{i}=L+h v_{i}$, which is proportional to vehicle speed and can become very large when the vehicle travels at high speed.

Adding the time headway term $\left(h v_{i}\right)$ improves the stability. This improvement is not due to enlarging inter-vehicle distance, but to the fact that it is a function of the velocity. So, the main idea of this paper is to propose a novel spacing error defining the time headway term proportional to the difference between the velocity of the vehicle and some value $V$ shared between all other vehicles in the platoon. We will discuss later how to set the parameter $V$. In this case, we define the novel error as:

$$
\delta_{i}=e_{i}-h\left(v_{i}-V\right)=\Delta X_{i}-L-h\left(v_{i}-V\right)
$$

where $V$ is the same velocity value shared between all the vehicles at the same sampling time.

The new control law is defined by : 


$$
u_{i}=\frac{\dot{e}_{i}+\lambda \delta_{i}}{h}
$$

which is represented in figure (5) for the i-th vehicle.

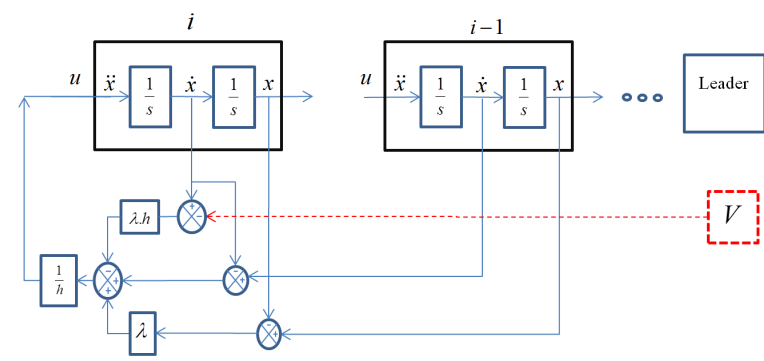

Figure 5: Control scheme of the i-th vehicle

The dynamic error becomes :

$$
h \ddot{e}_{i}+(1+\lambda h) \dot{e}_{i}+\lambda e_{i}=\dot{e}_{i-1}+\lambda e_{i-1}
$$

For the first car, we have:

$$
h \ddot{e}_{1}+\dot{e}_{1}+\lambda e_{1}=h \ddot{x}_{L}+\lambda\left(\dot{x}_{1}-V\right)
$$

where $\ddot{x}_{L}$ is leader acceleration.

Then, at equilibrium it comes :

$$
e_{i}=e_{i-1}, i=1 \ldots N
$$

and

$$
e_{1}=h \ddot{x}_{L}+\lambda\left(\dot{x}_{1}-V\right)=h u_{L}+\lambda\left(\dot{x}_{1}-V\right)
$$

where $u_{L}$ is the leader input.

So we get :

$$
e_{1}=e_{2}=\ldots=e_{N}=h u_{L}+\lambda\left(\dot{x}_{1}-V\right)
$$

To verify the effectiveness of the new law, the string stability of the platoon and the robustness of this control law must be analyzed.

\subsection{String Stability Analysis}

General string stability definition is given in (Swaroop, 1997), which means that, all the states are limited if the initial states (position and velocity errors) are limited and summable.

A sufficient condition for string stability is given in (Rajamani, 2006): $\left\|e_{i}\right\|_{\infty} \leq\left\|e_{i-1}\right\|_{\infty}$ which means that the spacing error must not increase as it propagates through the platoon. To verify this condition, the spacing error propagation transfer function is defined by :

$$
G_{i}(s)=\frac{e_{i}(s)}{e_{i-1}(s)}
$$

A sufficient condition for string stability is given by :

$$
\left\|G_{i}(s)\right\|_{\infty} \leq 1 \quad \text { and } \quad g_{i}(t)>0 \quad i=1,2 . . N
$$

where $g_{i}(t)$ is the error propagation impulse response of the i-th vehicle.

So to verify the string stability of a platoon using the novel spacing error, the spacing error propagation transfer function $H(s)$ must be computed :

$$
G_{i}(s)=\frac{e_{i}(s)}{e_{i-1}(s)}=\frac{1}{h \cdot s+1}
$$

After some development, it can be shown that :

$$
\left\{\begin{array}{l}
\left|G_{i}(\omega)\right|=\frac{1}{\sqrt{h^{2} \omega^{2}+1}} \leq 1 \\
g_{i}(t)=\frac{e^{\frac{-t}{h}}}{h}>0
\end{array}\right.
$$

In other words, at all frequencies, the spacing errors do not increase as they propagate through the platoon, so the system is string stable.

\subsection{Robustness to Unmodeled Actuation and Sensing Lags}

All realistic systems have actuation and sensing lags. Usual approximate models of the non linear actuation systems ignore some actuation dynamics, this unmodeled dynamics may produce lag in the actuator response. Sensing lags is due to the sampling time or filtering time. So we have to take the problem of the lag into consideration and verify the robustness of the control system to unmodeled actuation and sensing lags, which can be taken in consideration as follows (Swaroop and Rajagopal, 2001):

$$
\begin{aligned}
& \ddot{x}_{i}=u_{c, i} \\
& \tau \dot{u}_{c, i}+u_{c, i}=u_{i}
\end{aligned}
$$

where $\tau$ is a small unknown constant which represents lag time constant, $u_{c, i}$ is the real acceleration of the $\mathrm{i}$ th vehicle, and $u_{i}$ is the control law. All the sensing and actuation lags in the system have been modeled in the second equation in an aggregate manner.

The new spacing error propagation transfer function becomes:

$$
G_{p}(s)=\frac{e_{i}(s)}{e_{i-1}(s)}
$$

$$
G_{p}(s)=\frac{s+\lambda}{\tau h s^{3}+h s^{2}+(1+\lambda \cdot h) s+\lambda}
$$


The subscript $\mathrm{p}$ in the transfer function $H_{p}(s)$ has been used to indicate that it describes the error propagation transfer function for the disturbed system. To assure the string stability for this disturbed system, $\left|H_{p}(j . \omega)\right| \leq 1$ for all $\omega$ must be verified. So it comes :

$$
\omega^{2}+\lambda^{2} \leq\left(\lambda-h \omega^{2}\right)^{2}+\left(1+\lambda h-\tau h \omega^{2}\right)^{2} \omega^{2}
$$

which is equivalent to :

$$
\tau^{2} h^{2} \omega^{4}+\left(h^{2}-2 \tau h(1+\lambda h)\right) \omega^{2}+\lambda^{2} h^{2} \geq 0
$$

The above inequality holds only if the discriminant is negative or if the coefficient of $\omega^{2}$ is positive. Both conditions are satisfied (Swaroop, 1997) only if

$$
\tau \leq \frac{h}{2}
$$

This means the sum of all unmodeled lags must be less than or equal to half the time headway employed.

\subsection{Unmodeled vehicle motor and static spacing error}

Equation 7 shows that the static spacing error is the same for all the vehicles, and it is equal to the leader input $u_{L}$ multiplied by $h$, in addition to $\lambda .\left(\dot{x}_{1}-V\right)$. The second term, as it will become equal to zero at equilibrium, will be ignored. So the steady state error becomes:

$$
e_{1}=e_{2}=\ldots=e_{N}=h u_{L}
$$

At equilibrium all the vehicles have the same velocity $v_{d}$. In this case the input $u_{L}$ of the double integrator model of the leader, $\ddot{x}_{L}=u_{L}$, must be zero, hence the steady state spacing error will become equal to zero.

But as the motor model has not been taken into account, the actual car model will be not equivalent strickly to a double integrator. For instance, if the model for vehicle velocity is given by :

$$
\frac{V_{L}(s)}{u_{L}(s)}=\frac{K_{m}}{1+T_{m} s}
$$

in this case the system input will not be zero at equilibrium. It will be equal to $u_{L}=\frac{V_{d}}{K_{m}}$, so the spacing error will be given by the following:

$$
e_{1}=e_{2}=\ldots=e_{N}=\frac{h V_{d}}{\lambda K_{m}}
$$

Obviously, the model of the motor must be defined and take into account. It was out of scope of this paper.

\section{SIMULATIONS}

The control law have been checked under Matlab, and using TORCS to get more realistic results (as it takes more phenomena into account) and to have visual output when applying the novel spacing error.

All the simulations will be done on a straight road in Matlab and with small curvatures in TORCS. The desired speed of the leader of the platoon is changed three times (see figure 6) to check the transit response and the stability of the platoon. The desired intervehicle distance (bumper-to bumper distance, so we omit all the cars lengths from all following figures) is fixed to $L=5 \mathrm{~m}$. We have chosen $\lambda=1, h=1$ which give good compromise between stability and inter-vehicle distance.

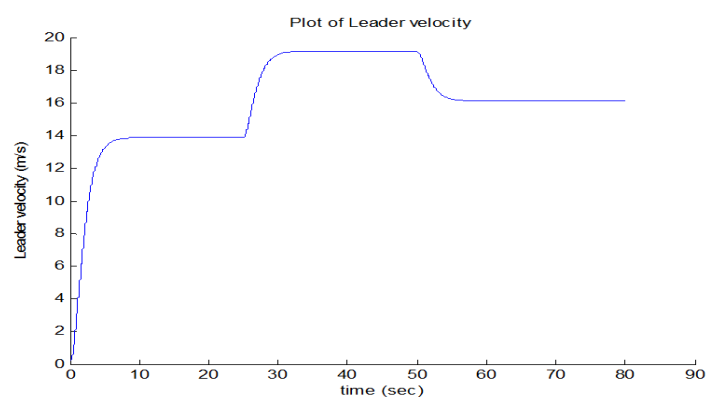

Figure 6: Leader's velocity profile

\subsection{Matlab results}

The linearized car model given in equation (3) has been used in simulation, which is ideal situation to check the validity of the control law without any disturbances.

Figure 7 shows the reduction in inter-vehicle distance using the novel control law, compared to the classical time headway control law. Inter-vehicle distances are greatly reduced and become equal to the desired $L$ at equilibrium. In addition, to assure the platoon string stability during the dynamic changes, the desired distance changes. The system is string stable (the error $e_{i}$ decreases as i increases).

Then, robustness of the new control law by taking the actuation and sensing lags into account has been checked. A lag time equal to $\tau=0.25 s$ satisfying the condition given in equation (12) has been chosen, and the corresponding results are given by the figure 8 : the system is still string stable.

As said before, the car model used under Matlab is very simple (i.e. ideal model). To be more realistic and to take more physical phenomena, new simulation have done done using TORCS environment. 

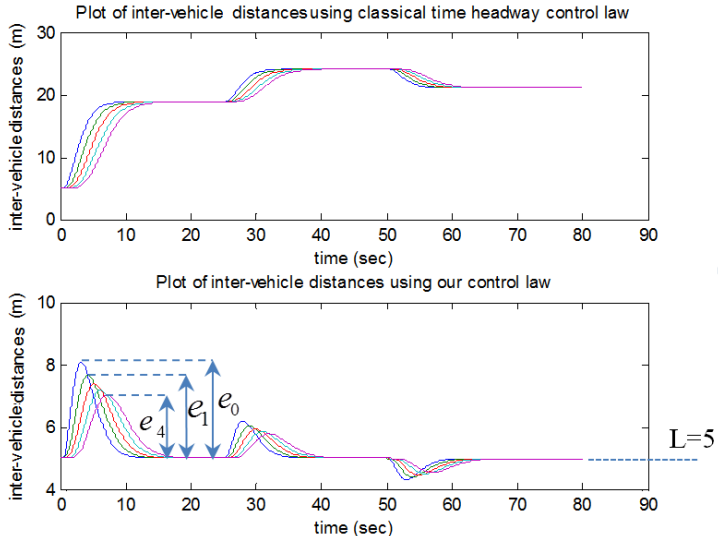

Figure 7: Inter-vehicle distance using CTH law and the new control law (in Matlab)

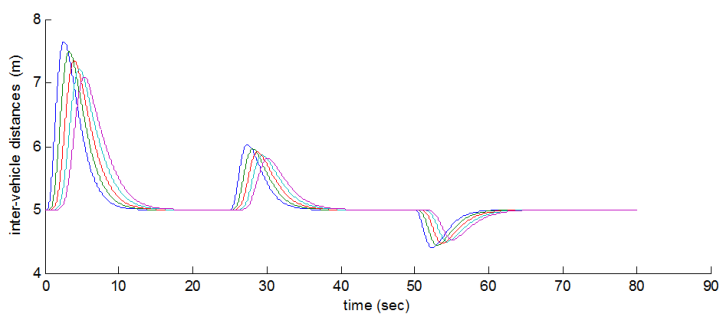

Figure 8: Inter-vehicle distance after taking lags into account

\subsection{TORCS results}

The Open Racing Car Simulator (Onieva et al., 2009) is one of the most popular car racing simulators. It is written in $\mathrm{C}++$ and is available under GPL license from its web page. TORCS presents several advantages for academic purposes, such as:

1. It lies between advanced simulators, like recent commercial car racing games, and a fully customizable environment, like the ones typically used by computational intelligence researchers for benchmark purposes.

2. It features a sophisticated physics engine (aerodynamics, fuel consumption, traction...) as well as a $3 \mathrm{D}$ graphics engine for the visualization of the races.

3. It was not conceived as a free alternative to commercial racing games, but it was specifically devised to make it as easy as possible to develop your own controller.

Same simulations as under Matlab have been performed, but using a road with small curvatures, taking the car model in equation (1), linearized using equation (2). As explain in the subsection 3.5, the motor model has not been taken into account in the novel control law.

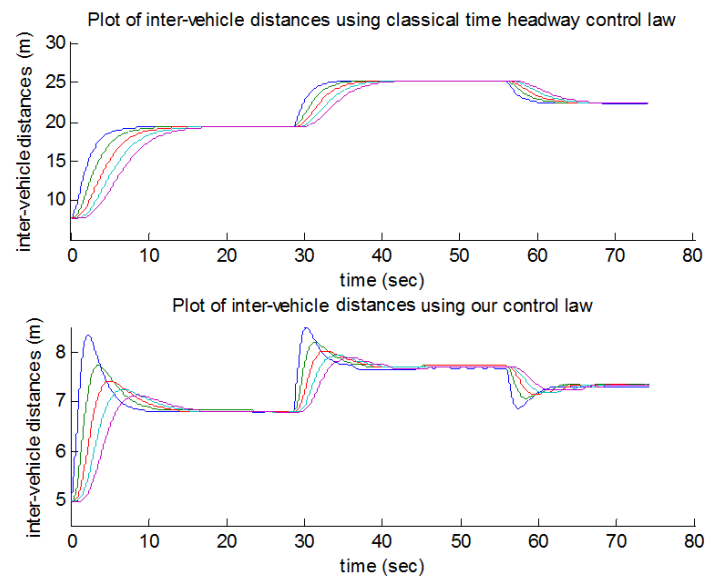

Figure 9: Inter-vehicle distances using CTH law and our law (in TORCS)

In figure 9, the same results have been obtained in TORCS as in Matlab concerning the stability and reduction of the inter-vehicle distances. It can be seen that the inter-vehicle distance at equilibrium isn't exactly equal to the desired distance $\mathrm{L}=5 \mathrm{~m}$, as it was explain in subsection 3.5.

In the same way, immunity of the control law to actuation and sensing lags has been evaluated: the platoon remains string stable even if actuation and sensing lags equal to $\tau=0.25 \mathrm{~s}$ have been added. The results are shown in figure 10 , where the system is string stable. So, the novel control is robust to lag errors.
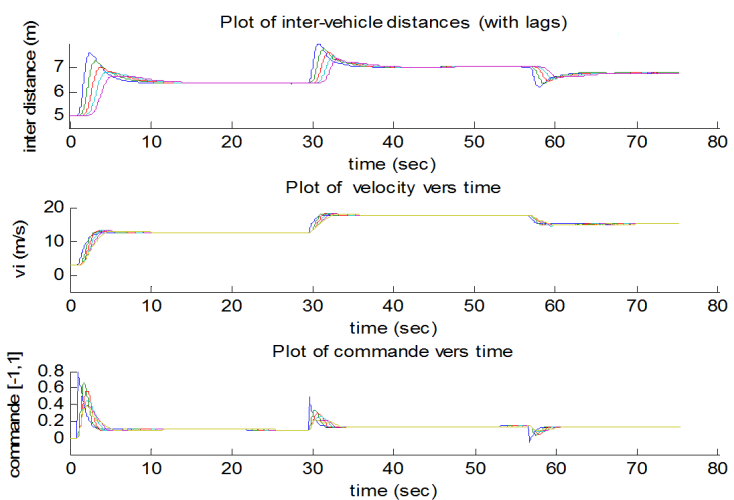

Figure 10: inter-vehicle distances after taking lags into account

Again we tried to check the immunity of the control law to actuation and sensing lags, we also found here that the platoon still string stable even if we add actuation and sensing lags equal to $\tau=0.25 \mathrm{~s}$. The 
results is shown in figure(10), where we can see that the system is string stable, so the control is robust to lag errors.

\section{DISCUSSION}

the proposed approach greatly reduces the intervehicle distances required to assure the stability. This is obtained by making the distance proportional, not to velocity, but to the difference between the vehicle velocity and a common velocity value shared by all vehicles of the platoon.

\subsection{Advantages and comparison}

Using the new spacing error and the corresponding new control law, the advantages are the following :

String stability: The propagation function $H(s)$, corresponding to the new control law, is not related to $V$, so its value will not affect the platoon stability. It can be noticed, that it is exactly the same propagation transfer function for the classical time headway spacing policy (Swaroop and Rajagopal, 2001; Yanakiev and Kanellakopoulos, 1996), so with this modification the system remains string stable.

Inter-vehicle distance: The most important effect of the proposed modification is on the inter-vehicle distances. At equilibrium it comes $\Delta X_{i}=L+h\left(v_{L}-V\right)$, by choosing $V=v_{L}$ the inter-vehicle distance becomes $\Delta X_{i}=L$, and during dynamic changes the inter-vehicle distance becomes $\Delta X_{i}=L+h\left(v_{i}-V_{L}\right)$.

The inter-vehicle distance has been decreased from $\Delta X_{i}=L+h v_{i}$ (which might be very large at high speeds) in the case of the classical time headway policy (Swaroop and Rajagopal, 2001; Xiao and Gao, 2011), to become $\Delta X_{i}=L+h\left(v_{i}-V\right)$ (which is exactly equal to $L$ at equilibrium and a little bit larger than it during transient phase). So during transient phase, the length of the platoon will be little different from the length of a platoon using constant spacing policy (Yanakiev and Kanellakopoulos, 1996; Swaroop, 1997).

Another important thing is the effect of increasing the $h$ parameter (which has many positive effects on stability...). In CTH it has a big negative effect on the inter-vehicle distance; as this distance increases proportionally to $h$, and hence the traffic density decreases. In our case, the inter distance is also proportional to $h$ but with a smaller coefficient $\left(v_{i}-V\right)$, so the inter distance changes will be smaller than the changes in CTH.
Collision: it is very clear that the possibility of collision between the vehicles is increased, as the inter distance between them is reduced. The problem of collision can be addressed separately from the problem of stability by adding a new term for the security. Communication: adding $V$ to the equation means that data must be exchanged. The amount of exchanged data between the vehicles by updating the value of $V$ every many sample times (depending on the rate of changes of the platoon velocity) can be reduced changing the value smoothly to avoid jumps in the inter distance.

Stability without communication: The string stability can be preserved even if the communication with the leader is totally lost, by switching to the classical time headway policy $V=0$ (full autonomous mode). In this case there is no need to communicate with the leader. So this law can keep the platoon stable even if communication is lost, on the contrary, it has been proved that the constant spacing policy can not be string stable, for homogeneous platoon with homogeneous control (all the gains are equals), without using any information from other vehicles (Seiler et al., 2004).

Hand checking protocol, between the leader and other vehicles, is very important to detect any loss of communication. If any loss is detected the leader will transmit an order to all vehicles to switch to full autonomous mode $V=0$, while the vehicle, which has lost the communication, will automatically switch to this mode.

Robustness: The condition for stability in the presence of lags $\tau$ has been found :

$$
h \geq 2 \tau
$$

It is difficult to increase $h$ in the classical time headway policy. But in the novel approach, it can be easily increased to accommodate the instability produced even by large lags, without large changes to the intervehicle distance. On the other hand, some simple constant spacing laws are not totally robust or only stable for small values of lags (Swaroop, 1997).

Control torque saturation: The control effort is inversely proportional to the desired headway time $h$ (Swaroop and Rajagopal, 2001), so decreasing $h$ may saturate the brake and engine torques. For the classical time headway, it is not possible to increase $h$ very much, but in the novel approach, it can be increased: this will prevent the control effort saturation, with only slight increase in the inter-vehicle distance. Simplicity and type of required data: The new control has the same simplicity of CTH law. It use the same variables of CTH in addition to a low rate updating of common speed (may be the leader or platoon speed). This last variable is the only difference 
with the classical time headway policy, while the constant spacing policy is always more complicated, as it may need the acceleration or other information, at least from the leader.

\subsection{Supervision of the parameter $V$}

As seen previously, the unique condition when adding $V$ to keep the platoon stable is to make it the same for all the vehicles at the same sample time. So, any value for $V$ (e.g. leader's velocity, the medium velocity of the platoon or the minimum velocity in the platoon...) can be chosen.

To increase the security and to prevent the collision, one can choose $V=\min \left(v_{\text {Leader }}, v_{1}, v_{2} \ldots, v_{N}\right)$. This will always make $h \cdot\left(v_{i}-V\right)>0$. In that case, the inter vehicle distance becomes $\Delta X_{i}=L+h\left(v_{i}-V\right)>$ $L$ but of course it will enlarge the inter-vehicle distance during velocity changes.

Changing $V$ quickly may has negative effect on the control and hence on the performance. So $V$ must not change quickly, or it must be interpolated to get smooth changes.

\section{CONCLUSIONS}

In this paper, the design of longitudinal control of platoons in highways has been addressed. A novel modification of CTH control law has been proposed in order to improve the large inter-vehicle distance (the main shortcoming of this control law). It also enables to increase the stability, the robustness, and avoid torque saturation. These benefits are obtained without the need for high rate data from other vehicles. Moreover, the platoon can still be stable even if the communication link is totally lost. All this results have been tested under Matlab and TORCS to check the validity of the novel approach.

This novel approach will enable us to apply the CTH law in real applications because the inter-vehicle distance becomes nearly equal to constant spacing policy.

\section{REFERENCES}

Franck, G., Vincent, C., and Francois, C. (2004). A reactive multi-agent system for localization and tracking in mobile robotics. In Tools with Artificial Intelligence, 2004. ICTAI 2004. 16th IEEE International Conference, pages $431-435$.

Huppe, X., de Lafontaine, J., Beauregard, M., and Michaud, F. (2003). Guidance and control of a platoon of vehicles adapted to changing environment conditions. In
Systems, Man and Cybernetics, 2003. IEEE International Conference, volume 4, pages 3091 - 3096 vol.4.

Ioannou, P. and Chien, C. (1993). Autonomous intelligent cruise control. Vehicular Technology, IEEE Transactions on, 42(4):657 -672.

Khatir, M. and Davison, E. (2004). Decentralized control of a large platoon of vehicles using non-identical controllers. In American Control Conference, 2004. Proceedings of the 2004, volume 3, pages $2769-2776$ vol.3.

Onieva, E., Pelta, D., Alonso, J., Milanes, V., and Perez, J. (2009). A modular parametric architecture for the torcs racing engine. In Computational Intelligence and Games, 2009. CIG 2009. IEEE Symposium on, pages $256-262$.

Rajamani, R. (2006). Vehicle dynamics and control. Springer Science, 1 edition.

Ricardo (2009). Cars that drive themselves can become reality within ten years. http://www.ricardo.com/enGB/News-Media/Press-releases/Newsreleases 1/2009/Cars-that-drive-themselves-canbecome-reality-within-ten-years/.

Royer, E., Bom, J., Dhome, M., Thuilot, B., Lhuillier, M., and Marmoiton, F. (2005). Outdoor autonomous navigation using monocular vision. In Intelligent Robots and Systems, 2005. (IROS 2005). 2005 IEEE/RSJ International Conference, pages 1253-1258.

Seiler, P., Pant, A., and Hedrick, K. (2004). Disturbance propagation in vehicle strings. Automatic Control, IEEE Transactions on, 49(10):1835 - 1842.

Sheikholeslam, S. and Desoer, C. A. (1989). Longitudinal control of a platoon of vehicles i: Linear model. Technical Report UCB/ERL M89/106, EECS Department, University of California, Berkeley.

Swaroop, D. (1997). String stability of interconnected systems: An application to platooning in automated highway systems. UC Berkeley: California Partners for Advanced Transit and Highways (PATH).

Swaroop, D. and Rajagopal, K. (2001). A review of constant time headway policy for automatic vehicle following. In Intelligent Transportation Systems, 2001. Proceedings. 2001 IEEE, pages $65-69$.

Thuilot, B. Bom, J. M. F. and Martinet, P. (2004). guidance of an urban electric vehicle relying on a kinematic gps sensor. In 5th IFAC Symposium on Intelligent Autonomous Vehicles (IAV04), Lisboa (Portugal), July 2004.

Xiao, L. and Gao, F. (2011). Practical string stability of platoon of adaptive cruise control vehicles. Intelligent Transportation Systems, IEEE Transactions on, 12(4):1184-1194.

Yanakiev, D. and Kanellakopoulos, I. (1995). Variable time headway for string stability of automated heavy-duty vehicles. In Proc. 34th IEEE CDC, pages 4077-4081.

Yanakiev, D. and Kanellakopoulos, I. (1996). A simplified framework for string stability analysis in ahs. In In Proceedings Of The 13th IFAC World Congress, pages 177-182. 